einfaches Veıbinden der Intensitätssprünge des Kathodenstrahles eingetragen. Um die extraterrestrische Intensität des Zodiakallichtes zu ermitteln, müssen natürlich die Korrekturen für Extinktion und Leuchten des Nachthimmels nachträglich angebracht werden.

Die Bestimmung des Polarisationsgrades und der Orientierung der Polarisationsebene des Zodiakallichtes geschieht mit Hilfe eines großen, ebenen Polaroidschirmes, der über den ganzen effektiven Querschnitt des Spiegels reicht und im parallelen Lichtbündel drehbar ist. Für diese Messungen wird der Spiegel nach einer gesuchten Gegend im Zodiakallicht ausgerichtet und in dieser Stellung festgehalten oder auch gegen die Erddrehung nachgeführt, wäh- rend der Polaroidschirm um gemessene Winkel gedreht wird. Die Meßwerte bestehen hier aus direkten Voltmeterablesungen.

Bisherige Resultate sind auf wenige Nächte beschränkt und können zum Hauptproblem, das hier gestellt ist, nämlich zur Untersuchung und Deutung der zeitlichen Schwankungen der Merkmale des Zodiakallichtes, noch nichts beitragen. Regelmäßige Messungen sollen im Herbst dieses Jahres aufgenommen werden.

Ein großer Teil der beschriebenen Arbeit ist von A. F. Beck, M. Cowan, R. Grenchik und J. D. G. Lindsay durchgeführt worden. Für die Bereitstellung von Mitteln sei der Research Corporation, New York, gedankt.

\title{
Aroser Messungen des Ozongehalts der unteren Troposphäre und sein Jahresgang*
}

\author{
Von F. W. Paul Götz und Friedrich Volz ${ }^{1}$ \\ Aus dem Lichtklimatischen Observatorium Arosa \\ (Z. Naturforschg. 6 a, 634-639 [1951]; eingegangen am 8. August 1951)
}

Erich Regener zum 70. Geburtstag

\begin{abstract}
Messungen des Ozongehalts in Arosa (1860 m ü. M.), durchgeführt seit März 1950 mit dem Gerät von Ehmert, werden diskutiert. Mit Ausnahme von Stau-Wetterlagen können die (Spitzen-)Werte als orographisch unbeeinflußt und damit für die freie Troposphäre repräsentativ angesehen werden. Auf der Rückseite von Kaltluftvorstößen werden in der Regel die höchsten Werte gemessen, in tropischer Luft die niedrigsten.

Es ergibt sich ein ausgesprochener Jahresgang: Dem geringsten Ozongehalt im Winter folgt rasche Auffüllung der Westdrift mit Ozon von Februar bis April (Maximalwert etwa $80 \gamma \mathrm{O}_{3} / \mathrm{m}^{3}$.

Die mit Dobson-Spektralphotometer gemessenen täglichen Werte des Gesamtozons über Arosa sind beigefügt.
\end{abstract}

$\mathrm{B}_{\mathrm{i}}^{\mathrm{e}}$ esonders bioklimatische Fragestellungen förderten in den letzten Jahren die Durchführung laufender Ozonmessungen in der Grundschicht; doch können diese Messungen wegen der raschen Zerstörung des Ozons in Bodennähe kein richtiges Bild des wetterhaften Ozongehalts der Luftmassen der Troposphäre geben. Über diesen sind bisher nur wenige, kurze Meßreihen - teils in den Alpen (Arosa ${ }^{2,3}$ und Jungfraujoch ${ }^{4,5}$ ), teils bei Flugzeugaufstiegen ${ }^{6,7}$ nach optischen und chemischen Methoden - durchgeführt wor-

\footnotetext{
* Diese Arbeit wurde mit Unterstützung der S c hwei zerischen Akademie der Medizinischen Wissen s ch a f t en, Basel, durchgeführt.

1 Mit frdl. Beihilfe von Fräulein Dr. G. P e r l.

2 F. W. P. Götz u. R. L a d e n b u rg, Naturwiss. 19, 373 [1931].

3 F. W. P. Götz u. R. P enndorf, Meteor. Z. 58, 409 [1941].
}

den. Die hier vorgebrachten wesentlichen Züge des Ozongehaltes der Troposphäre, und besonders sein Jahresgang, versprechen neue Einblicke in den Herabtransport des Ozons aus der Substratosphäre, den Ozonhaushalt in der Wettersphäre und damit den großräumigen Austausch und die planetarische Zirkulation, worauf schon eingehend E. R e g e n e r ${ }^{8}$ aufmerksam gemacht hat.

Vor allem ist zu untersuchen, inwieweit die in dem windgeschützten Aroser Hochtal — nördlich des Alpenhauptkammes - gewonnenen Ozonwerte re-

4 D. Chalonge, F. W. P. Götz u. E. Vassy, Naturwiss. 22, 297 [1934].

5 V. H. R e g e n e r, Meteor. Z. 55, 459 [1938].

6 A. E h m ert, Ber. deutsch. Wetterdienst US-Zone, Nr. 11 OZON, S. 63 (1949).

7 A. Ehm e r t 6 , S. 26.

8 E. R e ge n er, Meteor. Z. 60, 253 [1943]. 


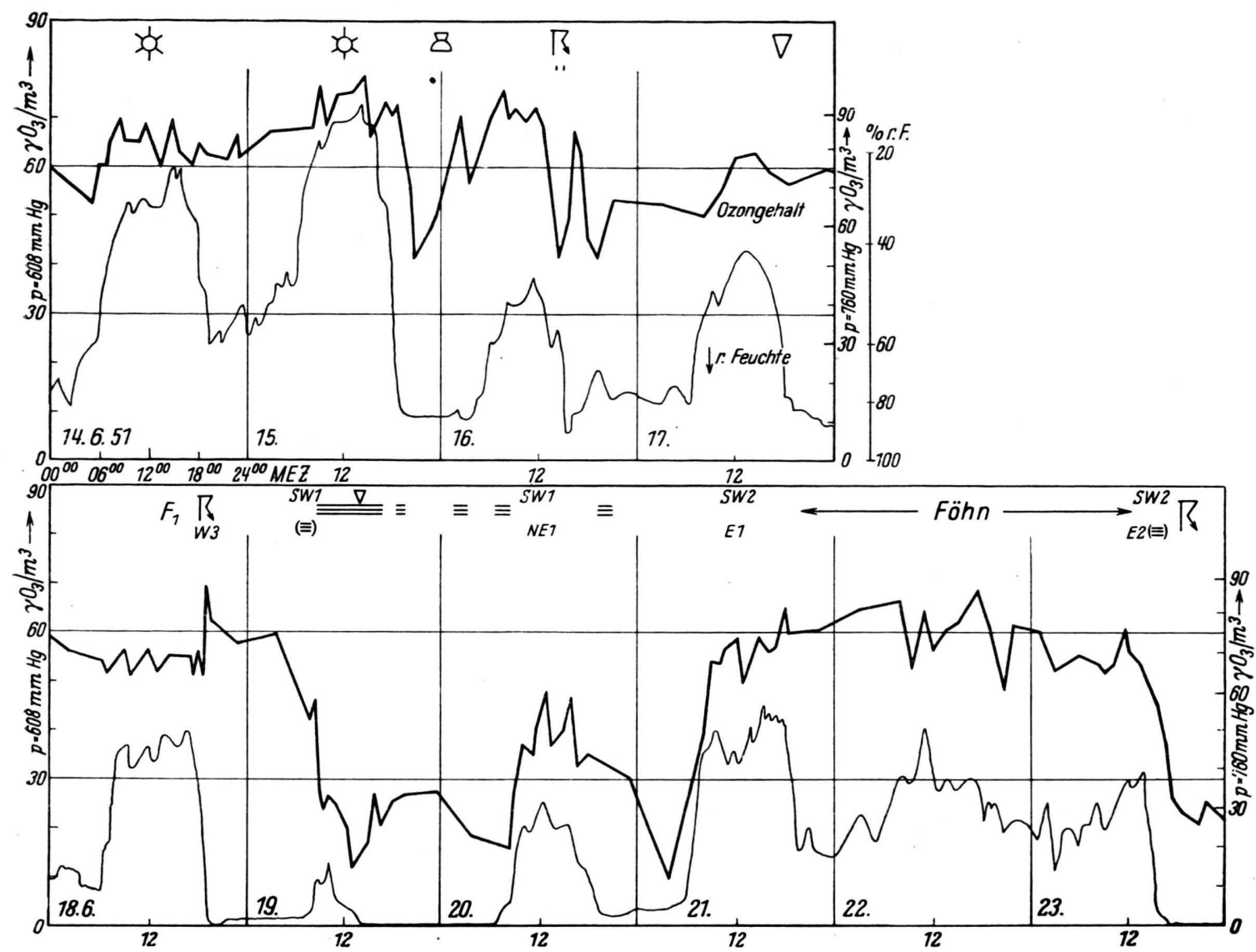

Abb. 1. Ozongehalt in Arosa bei typischen Wetterlagen. Ordinaten in $\gamma \mathrm{O}_{3} / \mathrm{m}^{3}$; links für Arosa, rechts auf Meereshöhe umgerechnet $\left(1 \gamma=10^{-6} \mathrm{~g}\right)$.

präsentativ sind, d. h. nicht örtlichen Einflüssen unterliegen. Zum Verständnis der orographischen Besonderheiten sei kurz erwähnt, daß das Observatorium ( $1860 \mathrm{~m}$ ü. M., etwa $140 \mathrm{~m}$ über dem Talgrund) an dem mäßig geneigten Südhang liegt, der die Nordseite einer flachen, ringsum von $2400-2900 \mathrm{~m}$ hohen Pässen und Bergzügen begrenzten „Bergschale“ ${ }^{9}$ bildet. Die Plessur verläßt mit relativ großem Gefälle die Bergschale gegen NE und eilt später dem Rhein in westlicher Richtung zu.

\section{Der Ozongehalt bei verschiedenen Wetterlagen}

Aus der kontinuierlichen Meßreihe, beginnend im März 1950, bringt die Abb. 1 einen Ausschnitt mit einigen für Arosa typischen Wetterabläufen. Die nach der elektrochemischen Methode von $\mathrm{E} \mathrm{h} \mathrm{m} \mathrm{e} \mathrm{r} \mathrm{t}{ }^{10}$ tags-

9 F. W. P. G ötz, Das Strahlungsklima von Arosa. Springer, Berlin 1926.

10 A. E h m e r t, Z. Naturforschg. 4 a, 321 [1949]. über etwa stündlich erhaltenen Werte des Ozongehalts sind in dem luftdruckabhängigen $\mathrm{Ma} \beta \gamma \mathrm{O}_{3} / \mathrm{m}^{3}$ aufgetragen (Arosa $608 \mathrm{~mm} \mathrm{Hg}$ im Mittel); die Skala des rechten Randes der Abbildungen gibt zum leichteren Vergleich mit den Messungen im Tiefland den auf Meereshöhe reduzierten Ozongehalt. Die Ozontagesgänge sind in den Abbildungen noch durch den spiegelbildlichen Verlauf der relativen Luftfeuchtigkeit ergänzt; die Tendenz und auch die Werte dieser "Lufttrockenheit" erwiesen sich bei der Bearbeitung als wertvoll, da sie den Austausch und die Luftströmung im Tal (Stau und Absinken) übersichtlich wiedergeben. - In der Zeile der Wettersymbole deuten die unteren Zeichen die meteorologischen Vorgänge im Tal an.

Ein erstes Beispiel vom 14. bis 23. Juni begann mit zwei Schönwettertagen. Am Vortag hatte eine Kaltfront Europa überquert, ein flaches Zwischenhoch lag nun über den Alpen. Die wenig schwankenden, hohen Ozonwerte entsprechen unserer Anschauung über die 
Windsysteme in Bergtälern. Während der Talaufwind allein ozonärmere Luft des Alpenvorlandes und Rheintals in den oberen Talabschnitt führen würde, sorgt das überlagerte Hangwindsystem für ihren Ersatz durch aus Kammhöhe nachströmende troposphärische Luft (s. a. ${ }^{11}$ ). Der nächtliche Bergwind bringt häufig dieselben Ozonwerte, wie sie auch tagsüber gemessen werden. Die Bildung eines nächtlichen Kaltluftsees ist in den verhältnismäßig steilen Tal nicht möglich. Am 15. Juni, dem zweiten „Strahlungstag“ mit deutlichem Absinken, brachte eine Luftschicht merklich höheren Ozongehalts den Jahreshöchstwert. Man hat bei „freiem Föhn“ im Hochgebirge natürlich die Möglichkeit, in zeitlicher Folge den Ozongehalt von Luftschichten zu messen, die ursprünglich einige km höher lagen, denn das Absinken hat im Hochdruckgebiet oft die Größenordnung von $1 \mathrm{~km}$ pro Tag. In unserem Beispiel war aber auch Advektion beteiligt, denn nach Abzug des Zwischenhochs passierte in der nächsten Nacht eine nur noch an ihrem Wolkenfeld erkenntliche Kaltfront die Alpen.

Am Vormittag des 16. Juni war der Ozongehalt wieder hoch, fiel aber nach $12 \mathrm{Uhr}$ rasch ab. Schon vorher war E-Wind notiert; auch der Feuchteverlauf zeigte an, daß Luft aus dem Tal heraufgeführt wurde. Um 14 Uhr waren zwei Gewitter, mit denen wohl die Stauerscheinung zusammenhing. Die Gewitter selbst haben aber, wie sich immer gezeigt hat, keinen Einfluß auf den Ozongang.

Am 17. Juni wurde in Kammhöhe leichte SW-Strömung beobachtet; abends kam es zu einem Regenschauer.

Am Vormittag des 18. setzte sich auch im Tal leichter Föhn $\left(F_{1}\right.$ in Abb. 1) durch, wie die Unruhe der Feuchteregistrierung bestätigte. Der Ozongehalt war fast konstant. - Infolge des Fallwindcharakters und der Böigkeit bewirkt der Föhn eine recht gute Durchmischung und raschen Ersatz der Luft in der Bergschale, so daß die gemessenen Ozonwerte ohne weiteres der in Kammhöhe strömenden Luft zugeordnet werden können. Oft bleibt bei Föhn der Ozongehalt bemerkenswert konstant, doch werden gelegentlich auch auffällige Schwankungen im Betrag von etwa 20 bis $30 \%$ gefunden, mit „Perioden“ voin 2 bis 3 Stdn. - Gegen Abend des 18.6. drehte der noch weiter zunehmende Wind auf West. Schon um 17 Uhr setzte Abkühlung und Feuchtezunahme ein; nach $18 \mathrm{Uhr}$ begann starker Schauerregen mit elektrischen Entladungen - die Gewitterfront überquerte

$11 \mathrm{H}$. Un geh e u e r, Ber. deutsch. Wetterdienst USZone, Nr. 12, S. 221 (1950). die ganzen Westalpen - , doch erst als der starke, von der Kammhöhe herabkommende Westwind abflaute und die Temperatur um $5^{\circ}$ sank, stieg das bis dahin fast konstante Ozon kurzzeitig um über $25 \%$ an. Bei leichtem NW-Wind blieb auch in der Nacht das Ozon etwas höher. Am Morgen des 19.6. war in Kammhöhe wieder leichter SW-Wind, während im Talgrund die für Kaltlufteinbrüche sehr charakteristische Nebelschlange („Churer Express“), erst schwach ausgebildet, langsam talaufwärts zog. Ab 9 Uhr bis gegen Abend war aber das ganze Tal mit dichtem Nebel erfüllt, der rasch bergwärts strömte, wobei der Ozongehalt ungewöhnlich nieder war. Die Kaltfront hatte vormittags die Alpen erreicht (um $10 \mathrm{Uhr}$ in Arosa schwacher Anstieg des Luftdrucks); die Hauptmasse der Kaltluft strömte nun über die Alpen, doch im Aroser Tal zog eine Luftschicht aufwärts, die wohl auch im Unterland sehr bodennah und deshalb ozonarm war. Es ist jedoch anzunehmen, daß die freiströmende Kaltluft normalen Ozongehalt hat, denn gelegentlich werden solche höheren Werte auch bei derartigen Staulagen gemessen. Vergleichsmessungen im Rheintal und auf einem freien Berggipfel sind besonders zur Prüfung dieser Annahmen (und neuer Erkenntnisse über die Ozonzerstörung) beabsichtigt.

Während des vor einer Kaltfront auf NW zurückdrehenden und zusammenbrechenden Föhns wurden schon oft auffällige Ozonanstiege gemessen (ähnlich dem vom 18.6. abends; s. auch in Abb. 2 am 5.7. 1950), sofern nicht wie am 23. 6. das Aufwärtsströmen der ozonarmen Talluft einsetzte, bevor die ozonreichere (voreilende) „Höhenkaltluft“ fallwindartig ins Tal herabstürzen konnte. Am 20.6. brach zwar die Bewölkung auf, doch war im Tal immer noch NEWind; an den Bergkämmen ging die Luft unter starker Quellwolkenbildung in die in der Höhe schon wieder herrschende SW-Strömung über. Erst am folgenden 21. Juni, nachdem sich stärkeres Absinken durchgesetzt hatte, stieg das Ozon wieder auf einen hohen Wert an. Es wurde zunehmend föhnig. Vom 22. auf den 23.6. nahm der (mittlere) Ozongehalt wieder deutlich um etwa $15 \%$ ab - es war aber noch immer die arktische Kaltluft, welche am 20.6. nach Süden (Atlantik) ausgebrochen war, und nun nach dem Weg über Spanien, erwärmt und ozonärmer, „zurückkehrte“.

Abermals näherten sich von Westen Kaltfronten, doch diesmal begann schon 6 Stdn. vor Ankunft der Gewitterfront die ozonarme Luftströmung talaufwärts, während in Kammhöhe noch immer starker SW-Wind war. Wieder hielt die Staulage mehrere Tage an. 
Ein interessanter Wechsel des Ozongehalts in Luftmassen, die von südlicher Höhenströmung herangeführt wurden, ließ sich anfangs Juli 1950 verfolgen; der Ozongang ist in Abb. 2 dargestellt.

Am Nachmittag des 4. 7. nahm plötzlich bei schwachem Föhn der Ozongehalt der Luft sehr auffällig $a b$; bis zum nächsten Mittag stieg er wieder etwas an. Der Föhn hatte inzwischen beträchtliche Stärke erreicht: an der Vorderseite des in Arosa in der Nacht zum 6. 7. erfolgenden Kaltlufteinbruchs (der ebenso ablief wie derjenige am 18.6.51!) wurde subtropische Luft über die Alpen transportiert. - Am 1.7. war eine Kaltfront vom Atlantik her auf das afrikanische Festland übergetreten. In die zugehörige Kaltluft, welche nun über dem westlichen Marokko lag, lassen

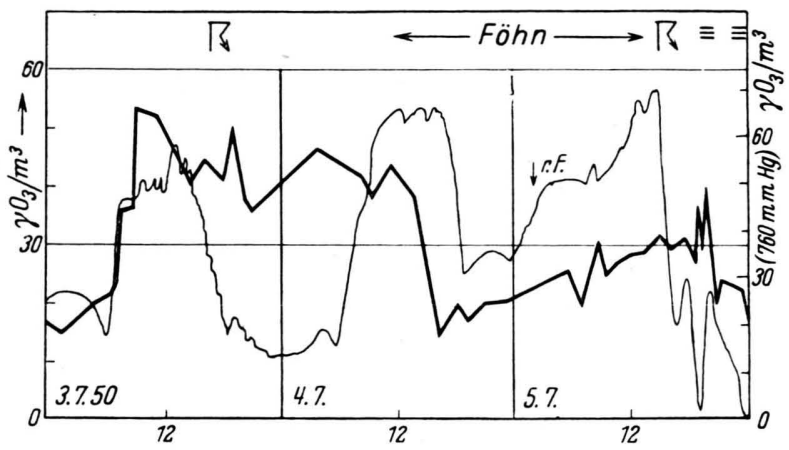

Abb. 2. Ozongehalt in Luftmassen verschiedener Herkunft.

sich die Trajektorien (der 700-mb-Fläche) der am 3.7. nachmittags und in der Frühe des nächsten Tages die Alpen überquerenden Luftmassen normalen Ozongehaltes zurückverfolgen, während es sich aus den Höhenwetterkarten ergibt, daß die ozonarmen Luftmassen die algerische Küste etwa einen Tag, bevor sie die Alpen (4. auf 5. 7.50) erreichten, passiert hatten; die Kaltfront lag aber noch weit westlich. Es kann (auch zufolge der Bodenwetterkarte dieser Tage) wohl angenommen werden, daß die unteren Luftschichten des Mittelmeergebietes nicht wesentlich in die Advektion dieses Scirocco einbezogen waren. Diese Luft wird schon einige Zeit über dem afrikanischen Kontinent gewesen sein.

Auch die Luftmassen, die am 2. und 3. 7. recht niederen Ozongehalt (rund $20 \gamma \mathrm{O}_{3} / \mathrm{m}^{3}$ ) aufwiesen, scheinen sich zuvor in windschwachen Hochdruckgebieten des Mittelmeerraumes aufgehalten zu haben.

Auffällig niedrige Werte des Ozongehaltes traten vom 19. bis 24. Oktober 1950 während einer Schönwetterperiode mit starkem Absinken auf (s.
Jahresgang Abb.3). Die Luftbahnen für den 20. 10 . ließen sich in die Nähe des Kernes des Azorenhochs zurückverfolgen. Am nächsten Tag wurden bei starkem Absinken der Luft über $12 \mathrm{Stdn}$. lang nur $8 \gamma \mathrm{O}_{3} / \mathrm{m}^{3}$ gemessen - der tiefste "repräsentative“ Wert, der bisher in Arosa auftrat. Doch fand $\mathrm{Ehmert}{ }^{6}$ bei Flugzeugaufstiegen in einem winterlichen Hoch bis über $4 \mathrm{~km}$ Höhe einen ähnlich niederen Ozongehalt.

$$
\text { Der Jahresgang des Ozongehalts }
$$

Aus unseren nun $1^{1 / 2}$ Jahre umfassenden Messungen ergibt sich ein ausgesprochener Jahresgang des Ozongehalts der unteren Troposphäre. In der Abb. 3 sind die täglichen Spitzenwerte von April 1950 bis März 1951 eingetragen, sofern sie nicht als orographisch (Stau) beeinflußt anzusehen waren. - Im Vergleich zu unseren Ergebnissen mißt man in der Grundschicht, wo die vertikale Turbulenz für den Gehalt an „bodennahem“ Ozon ausschlaggebend ist, im Mittel kaum halb so hohe Spitzenwerte (auf gleichen Luftdruck bezogen). Im Zeitraum Mai bis Juli 1950 wurden z. B. an einem Ort der Rheinebene nur 12 gleichhohe Tages-Spitzenwerte gemessen, und an einem anderen Ort stärkerer Tallage erreichten von April bis September 1950 nur rund 7 Spitzenwerte etwa 75\% des Wertes, der in Arosa an demselben Tag (Staulagen ausgenommen) erhalten wurde. Es ist also nicht möglich, aus den Messungen in der Grundschicht direkt auf den Ozongehalt von troposphärischen Luftmassen zu schließen.

Die wesentlichen Züge des Jahresganges lassen sich, einer eingehenderen Darstellung vorgreifend, kurz skizzieren:

Im Herbst und Winter scheint die arktische Ozonquelle ziemlich versiegt zu sein, der mittlere Ozongehalt der Troposphäre ist recht gering; im Gefolge von Advektion arktischer Kaltluft treten im Winter aber gelegentlich relativ hohe Spitzenwerte auf. Erst im März jedoch schwillt in den gemäßigten Breiten auch der mittlere Ozongehalt kräftig an - inzwischen hat ja der Betrag des hohen Ozons, vor allem in nördlichen Breiten, seinen Maximalwert erlangt. Gegen Ende April, der Zeit häufiger, hochreichender Kaltluftausbrüche und des plötzlichen Anstiegs der mittleren Höhe der Tropopause ${ }^{12}$ hat die Speisung der Westdrift mit Ozon aus der substratosphärischen Quelle ihren Höhepunkt erreicht.

Die allgemeine Zirkulation des Sommers dürfte, nach den bisherigen Vergleichsmöglichkeiten zu schließen,

12 F. M ölle r, Meteor. Z. 55, 161 [1938]. 


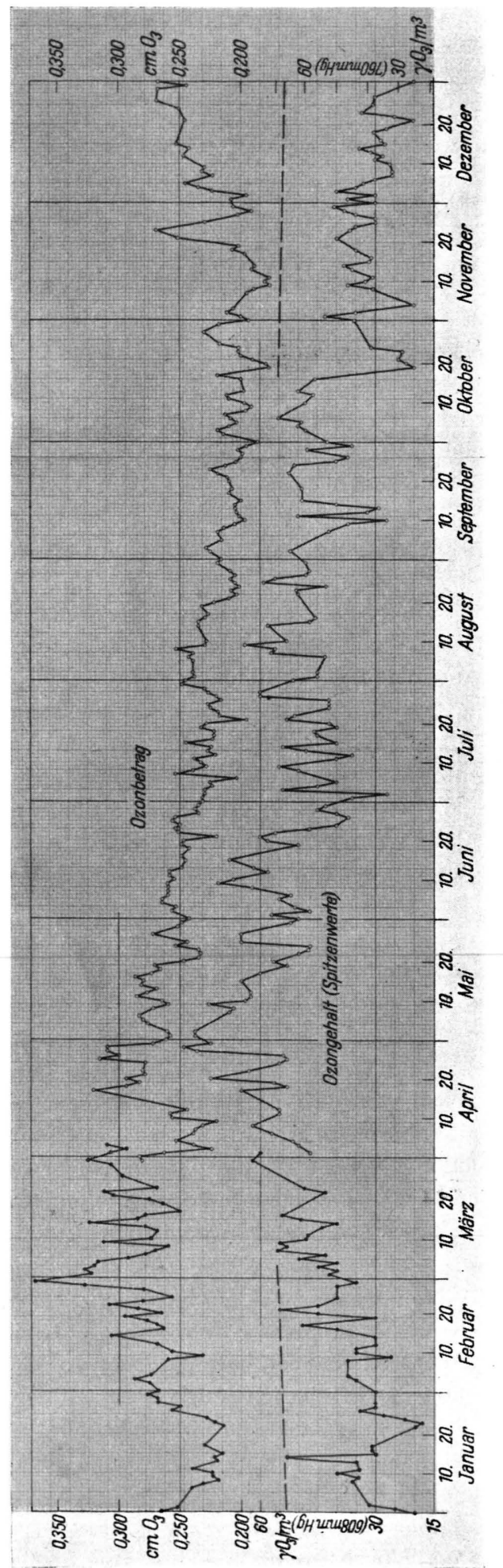

in den Ozonwerten gut zum Ausdruck kommen. So fallen in den beiden Jahren 1950 und (in Abb. 3 nicht wiedergegeben) 1951 zwei Gipfel der Spitzenwerte Ende April und Mitte Juni auf; ihre "Singularität" (Kältewellen!) müßte durch Fortführung der Messungen erwiesen werden. - Im Juni und Juli 1950, als (im Monatsmittel) der Subtropenhochgürtel etwas nach Norden verschoben war - entsprechend den übernormalen Mitteln der Temperatur in der ganzen Troposphäre - waren sowohl das Mittel des Ozongehalts (Abb.4) als auch die Spitzenwerte deutlich niedriger als bei südlicherer Lage der Frontalzone in der entsprechenden Zeit des Jahres 1951. Auch Einzelfälle, wie z. B. die auf S. 637 besprochenen Wetter-

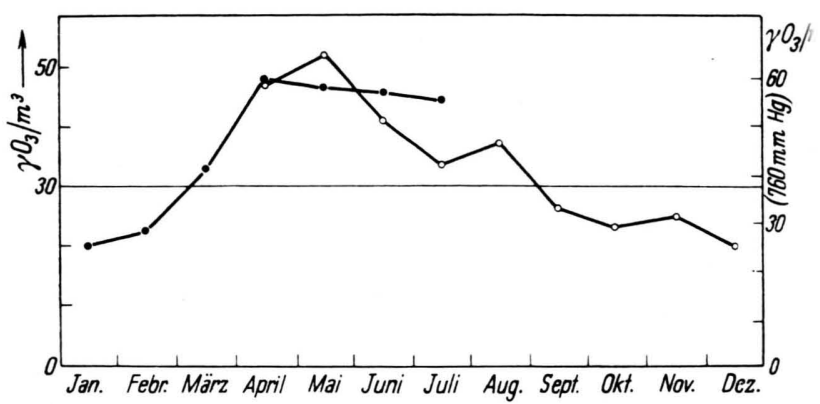

Abb. 4. Monatsmittel des Ozongehalts in Arosa $(\bullet \bullet \bullet 1950 ; \circ 0 \circ 1951)$.

lagen, bestätigen die bekannte Ansicht, daß in der Troposphäre ein starkes Ozongefälle gegen die Subtropen hin besteht.

Die Realität dieses Jahresganges wird dadurch wesentlich gesichert, daß die Extremwerte, welche an verschiedenen Orten, besonders auch in zurückliegenden Jahren (vgl. ${ }^{-7}, 13$ ), gemessen wurden, sich gut in dieses Ergebnis einfügen. Es ist danach allerdings zu erwarten, daß in den Wintermonaten normalerweise - im Winter 1950/51 trat in unserem Gebiet kaum eine Absinklage ein - etwas höhere Spitzenwerte des Ozongehalts (um $50 \gamma \mathrm{O}_{3} / \mathrm{m}^{3}$ ) vorkommen ${ }^{14}$ (oberes Winterniveau in Abb. 3 gestrichelt).

In Abb. 4 sind die aus den Tagesmitteln (aller Tage) berechneten Monatsmittel des Ozongehaltes von April 1950 bis Juli 1951 dargestellt.

Die Tagesmittel des Gesamtbetrags des Ozons über

13 Landeswetterd. Württ.-Hohenz., Bioklimat. Monatsübers. 1950/51. Herausgeg. v. Met. Amt Tübingen; Bad. Landeswetterd., Zehntagebericht Bioklima-Instit. Staufen, Freiburg 1950.

$14 \mathrm{~S}$. die inzwischen veröffentlichten Messungen in NeuMexiko: I. G. B ow en u. V. H. Re ge ner, J. Geophysic. Res. 56, 307 [1951]. 
Arosa (mit dem Dobson-Spektrophotometer gemessen) sind außer den Spitzenwerten des troposphärischen Ozons in Abb. 3 eingezeichnet.

Die hier gegebene knappe Darstellung der Meßergebnisse und der daraus resultierenden Folgerungen werden dem reichen Material nicht gerecht. Spätere Veröffentlichungen sollen diese ergänzen, so neben statistischer und aerologischer Bearbeitung eine eingehende Diskussion des Jahresganges und seiner Ursachen und auch des Ozonhaushalts der Troposphäre. Hierbei werden neue Einsichten über die Ozonzerstörung eine wesentliche Rolle spielen.

\section{$\mathrm{Zum} \mathrm{Ozonklima}$}

Nach den Messungen sind die Grundelemente des Ozonklimas im Hochgebirge ganz anders als im Tiefland: a) kein nennenswerter Tagesgang, nur kleine und unregelmäßige Schwankungen des verhältnismäßig sehr hohen Ozongehalts;

b) selbst über längere Witterungsperioden hinweg ist in der Regel der Ozongehalt recht gleichmäßig;

c) dafür hat Arosa bei den fast immer mit Kaltlufteinbruch verbundenen Stauwetterlagen sehr niedere Ozonwerte.

Deshalb scheint in solcher Höhenlage ein systematischer Vergleich des Ozonganges mit Wetterfühligkeitserscheinungen und sonstigen physiologischen Reaktionen zur Klärung der Frage, ob dem Ozon solche Wirkungen zukommen, recht aufschlußreich ${ }^{15}$.

15 F. W. P. Götz, Ann. Schweiz. Ges. Baln. Klimat. 40, 33 [1950].

\title{
Über die Erfassung der atmosphärischen Ozonverteilung durch die Benutzung von Mondfinsternissen*
}

\author{
Von Hans-Karl Paetzold \\ Aus der Forschungsstelle für Physik der Stratosphäre in der Max-Planck-Gesellschaft \\ in Weißenau \\ (Z. Naturforschg. 6 a, 639-648 [1951]; eingegangen am 10. Juli 1951)
}

\section{Herrn Professor Erich Regener zum 70. Geburtstag}

\begin{abstract}
Aus der gemessenen Helligkeitsverteilung im Spektralgebiet bei $6000 \AA$ auf dem verfinsterten Mond senkrecht zur Schattengrenze läßt sich die vertikale Verteilung des Ozons in der Atmosphäre ableiten. Je nach dem Positionswinkel, unter dem die Helligkeitsverteilung gemessen wird, ergibt sich, unabhängig von der Lage des Beobachtungsortes auf der Erde, die Ozonverteilung für verschiedene Breiten, was besonders im Hinblick auf die Erdgegenden wertvoll ist, für die nur wenige oder gar keine Bestimmungen der Ozonverteilung vorliegen. Die Grundlagen des Verfahrens werden entwickelt und geprüft und die Brauchbarkeit der Methode wird an vier bislang bei Mondfinsternissen vorgenommenen Messungen aufgezeigt. Die auf diesem Wege gewonnenen Ozonverteilungen entsprechen den auf direktem Wege bei Ballonaufstiegen erhaltenen Ergebnissen. Um möglichst jede Mondfinsternis ausnützen zu können, ist eine breitere, von mehreren geographisch verteilten Stellen ausgehende Beteiligung erforderlich. Dazu werden für die Durchführung und Auswertung der Messungen einige praktische Hinweise gegeben.
\end{abstract}

I n einer früheren Arbeit ${ }^{1}$ wurde gezeigt, daß aus geeigneten photometrischen Beobachtungen bei Mondfinsternissen die vertikale Verteilung ${ }^{2}$ des atmosphärischen Ozons in großen Höhen abgeleitet werden kann. Dazu wird im Maximum der Chappuis-Bande des

* Ein Auszug erscheint im Bericht der Tagung Geophysik, Brüssel 1951 .

1 H.-K. P a e t z old, Z. Naturforschg. 5a, 661 [1950]. Die Arbeit wird im folgenden mit (I) bezeichnet.

2 Fußnote 2 s. S. 640.

Ozons bei $6000 \AA$ die Beleuchtungsverteilung auf der verfinsterten Mondoberfläche senkrecht zur Schattengrenze gemessen. Denn das bei einer Finsternis in den Schattenraum eindringende Licht muß die Erdatmosphäre durchlaufen, so daß sich die selektiv absorbierende Ozonschicht und ihre Verteilung in einer entsprechenden Filterung dieses Lichtes auswirken müssen. Im folgenden soll gezeigt werden, daß aus solchen photometrischen Messungen bei Mondfinster- 